\title{
Numerical Solution of Electromagnetic Problem MHD in a Polar Coordinates
}

\author{
Ahmed M.J. Jassim \\ ahmed.m.j.jassim@uomosul.edu.iq \\ Ahmed S.J. Amen \\ ahmedsalar@ntu.edu.iq \\ Department of Mathematics \\ College of Computer Sciences \& Mathematics \\ University of Mosul, Mosul, Iraq
}

Received on: 23/09/2013

Accepted on: 12/02/2014

\begin{abstract}
In this paper, we studied a flow of fluid in a pipe under the effect of electromagnetic field in a polar coordinate, be done build a mathematical model which represented by system of two dimensional non-linear partial differential equations and we solved it by using Alternating Directions Implicit method (ADI) which is one of the finite differences method, and from the numerical solution indicated the behavior of temperature distribution inside the pipe, be done explication the influence of Rayleigh number and Prandtl number as well as the influence of Eckert number upon the behavior of temperature distribution are also done through the energy equation in polar coordinate, we arrived to steady state from unsteady state, we find the behavior of fluid flow inside the pipe and also we studied the influence of Hartmann number upon the behavior of fluid flow in pipe and all this done through the motion equation in polar coordinate.
\end{abstract}

Keywords: Numerical Solution, Polar Coordinates, MHD Problem.

$$
\begin{aligned}
& \text { الحل العددي لمسألة كهرومغناطيسية (MHD) في الإحداثيات القطبية }
\end{aligned}
$$

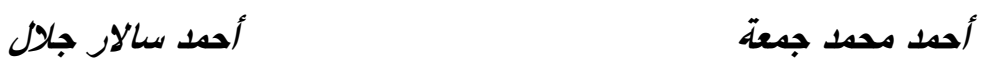

$$
\begin{aligned}
& \text { قسم الرياضيات، كلية علوم الحاسوب والرياضيات } \\
& \text { جامعة الموصل، الموصل، العرقاق }
\end{aligned}
$$

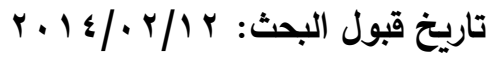

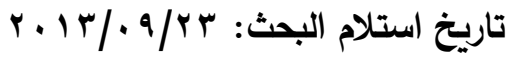


يعد موضوع حركة الموائع الممغنطة (MHD) (Magnetohydrodynamics) (أحد الفروع المهمة لميكانيك الموائع الذي يهتم بدراسة التفاعل الذي يجري بين سوائلِ وحقولِ كهرومغناطيسية عندما يتحرك

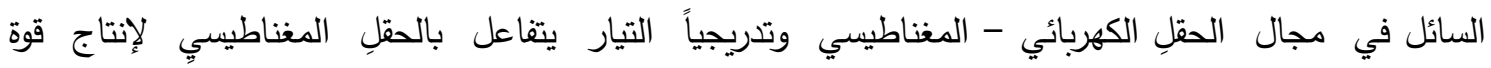

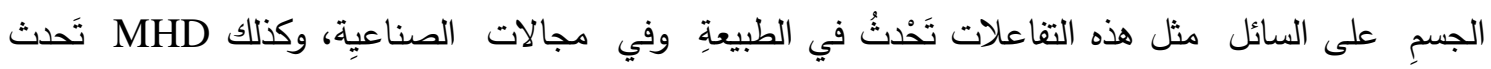

في الثمسِ وداخلِ الأرضِ وطبقات Ionosphere العليا والفضاء الخارجي [3]. ولذلك فإن هذا الموضوع قد جذب انتباه العديد من العلماء والباحثين، ففي عام 1968 حصل كل من Lykoudis و Gardner [7]. وفي عام 1999 درس Davidson ضبط الجريان الكهرومغناطيسي لفولاذ [2] وبعد ذلك قدم كل من فن فئساة

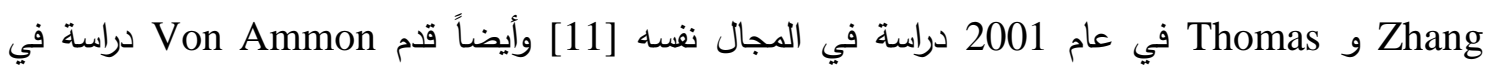

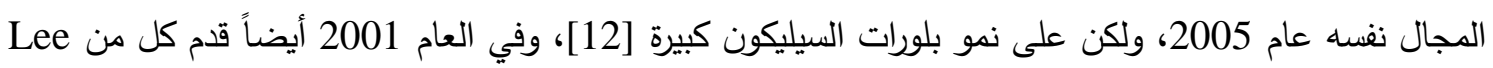
و Choi بحثاً وضحا فيه أنَّ الحقل المغناطيسي يمكن أن يؤدي إلى تخفيف كبير من عائق (موجود في قناة) أثناء

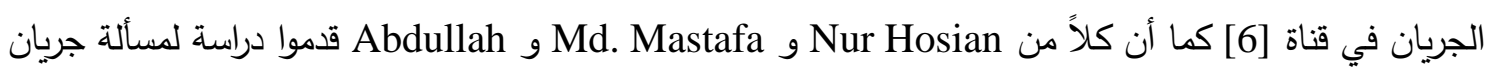
صفائحي حر بالحمل الحراري لمائع ممغنط (MHD) في أسطوانة أفقية [9]. وكذلك Ramamurthy أظهر توزيع السرعة والحقل المغناطيسي لمائع لزج غير مضغوط بين إدارة محورية لأسطوانة تحت تأثير حقل مغناطيسي

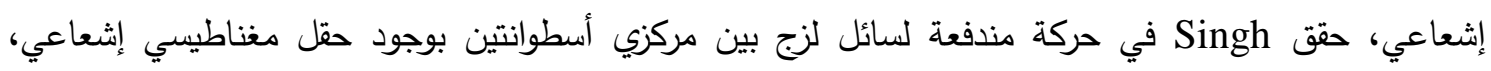

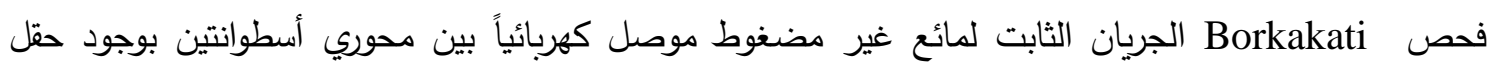

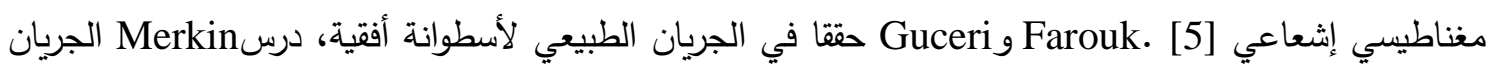
الطباقي حر انتقال للأسطوانة الدائرية المتساوية في درجة الحرارة.

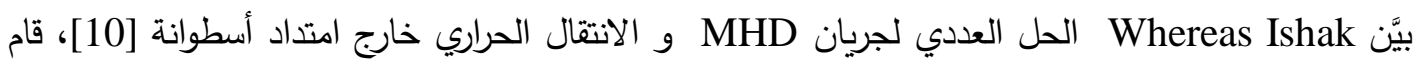
كل من Wumar و KatukaniوSparrow و بإلحاق مسألة جريان قرب قرص دائر إلى علم قوة الموائع (Hydrodynamic)، و درسوا جريان MHD قرب قرص دائر، درس Ariel تحليل الحاسوبي لجريان قرب قرص دائر [1]، حلGold MHD LoوGardner

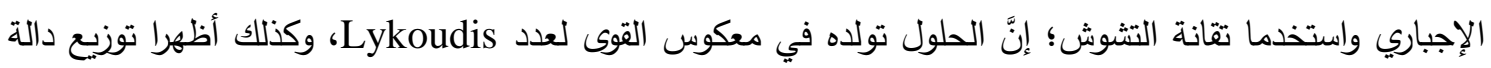
الجريان وسرعة سمتي (azimuthal) لبعض أعداد هارتمان التي تكون صغيرة [7]. وفي البحث الحالي درس مائع

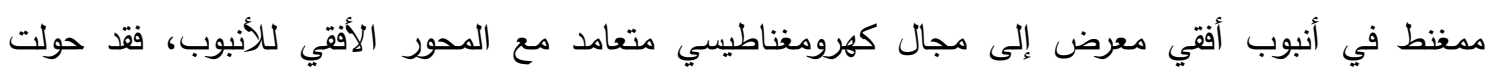
المعادلات التي تتحكم في المسألة إلى الإحداثيات القطبية وحلت عددياً بالاستخدام طريقة الاتجاهات المتعاقبة ضمنياً (ADI).

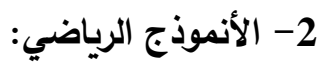
نقوم بدراسة جريان مائع موصل للكهربائية داخل أنبوب أفقي بعد تسليط مجال كهرومغناطيسي ثابت على مانى

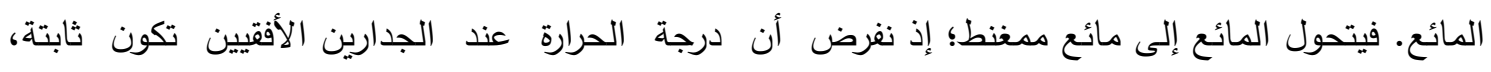

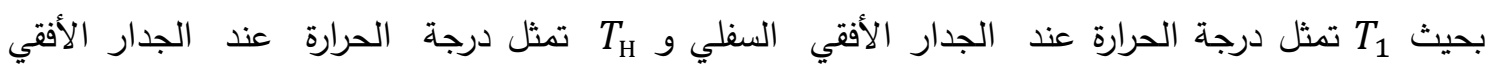




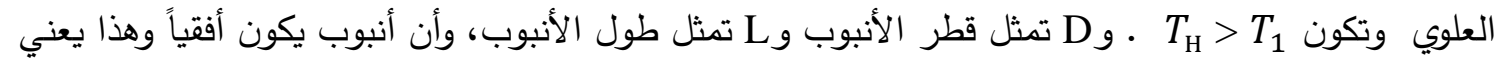

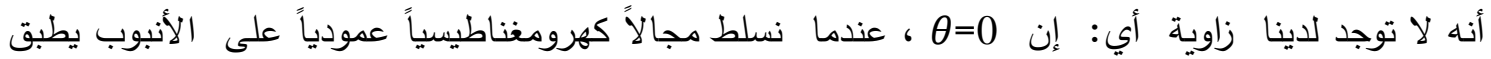
قاعدة الكف الأيمن عليه وكما يأتي: المجال الكهرومغناطيسي العمودي المسلط يمثل المحور ق، وبعد تسليط المجال الكهرومغناطيسي يتكون مائع ممغنط يجري باتجاه محور r وكذلك يتكون مجال كهرومغناطيسي مضاد باتجاه محور Z؛ إذ يكون مقداره صغيراً جداً يمكن إهماله. كما موضح في الثكل (1):

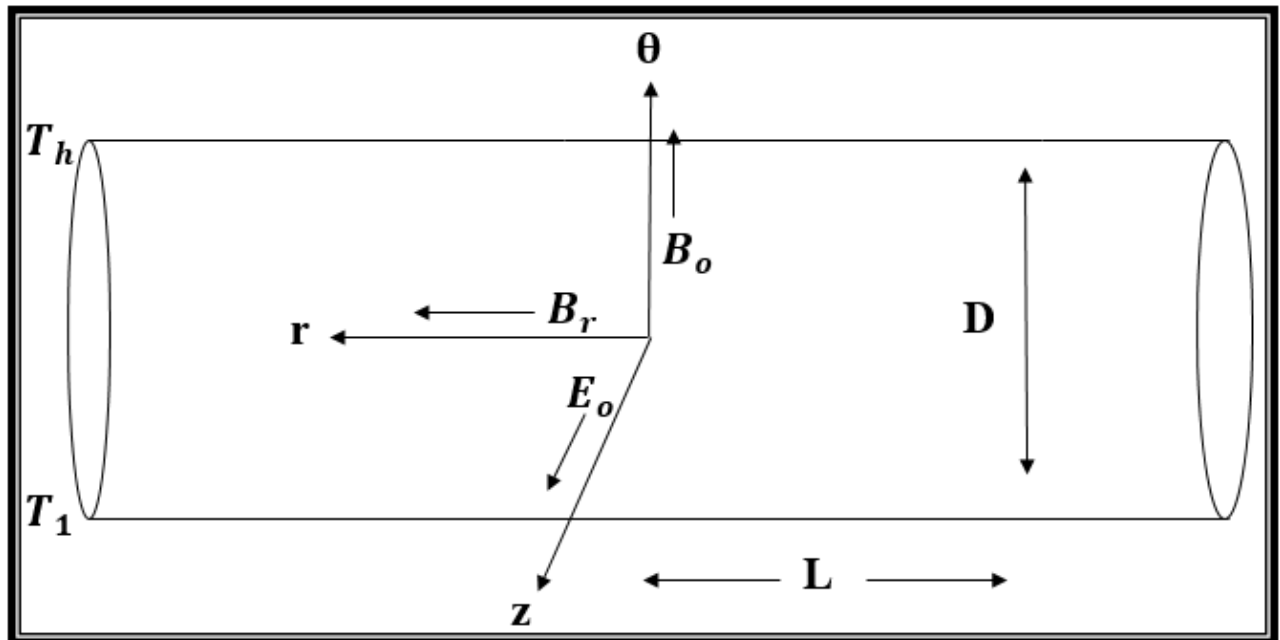

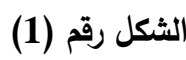

3- المعادلات التي تتحكم بالمسألة بالصيغة البعدية في الإحداثيات القطبية:

إن المعادلات المتحكمة بالمسألة بالصيغة البعدية هي:

$\frac{\partial v_{r}^{*}}{\partial \mathrm{r}^{*}}+\frac{v_{r}^{*}}{r^{*}}+\frac{\partial v_{\theta}^{*}}{r^{*} \partial \theta^{*}}=0$

$\rho\left[\frac{\partial v_{r}^{*}}{\partial t^{*}}+v_{\mathrm{r}}^{*} \frac{\partial v_{\mathrm{r}}^{*}}{\partial \mathrm{r}^{*}}+\frac{v_{\theta}^{*}}{\mathrm{r}^{*}} \frac{\partial v_{r}^{*}}{\partial \theta^{*}}-\frac{\left(v_{\theta}^{*}\right)^{2}}{r^{*}}\right]=-\frac{\partial p}{\partial r^{*}}+\mu\left[\frac{\partial^{2} v_{r}^{*}}{\partial r^{* 2}}+\frac{\partial v_{r}^{*}}{r^{*} \partial r^{*}}+\frac{1}{r^{* 2}} \frac{\partial^{2} v_{r}^{*}}{\partial \theta^{*^{2}}}-\right.$ $\left.\frac{v_{r}^{*}}{r^{2}}-\frac{2}{r^{*^{2}}} \frac{\partial v_{\theta}^{*}}{\partial \theta^{*}}\right]+\sigma E_{o} B_{o}+\sigma B_{o} E_{o} v_{r}^{*}-B_{r} B_{o} v_{\theta}^{*}$

$\rho\left[\frac{\partial v_{\theta}^{*}}{\partial t^{*}}+v_{\mathrm{r}}^{*} \frac{\partial v_{\theta}^{*}}{\partial \mathrm{r}^{*}}+\frac{v_{r}^{*} v_{\theta}^{*}}{r^{*}}+\frac{v_{\theta}^{*}}{r^{*}} \frac{\partial v_{\theta}^{*}}{\partial \theta^{*}}\right]=-\frac{\partial p}{r^{*} \partial \theta^{*}}+\mu\left[\frac{\partial^{2} v_{\theta}^{*}}{\partial r^{*^{2}}}+\frac{\partial v_{\theta}^{*}}{r^{*} \partial r^{*}}+\frac{2}{r^{* 2}} \frac{\partial v_{r}^{*}}{\partial \theta^{*}}-\right.$ $\left.\frac{v_{\theta}^{*}}{r^{*^{2}}}+\frac{1}{r^{*^{2}}} \frac{\partial^{2} v_{\theta}^{*}}{\partial \theta^{*^{2}}}\right]-\rho g \beta\left(\mathrm{T}-\mathrm{T}_{\mathrm{O}}\right)-\sigma B_{r} E_{o}-\sigma B_{r} B_{o} v_{r}^{*}+\sigma B_{r}^{2} v_{\theta}^{*}$

$\left[\frac{\partial \mathrm{T}}{\partial \mathrm{t}^{*}}+v_{r}^{*} \frac{\partial T}{\partial r^{*}}+\frac{v_{\theta}^{*}}{r^{*}} \frac{\partial T}{\partial \theta^{*}}\right]=\frac{k}{\rho \mathrm{c}_{\mathrm{p}}}\left[\frac{\partial^{2} T}{\partial \mathrm{r}^{*^{2}}}+\frac{\partial T}{r^{*} \partial r^{*}}+\frac{\partial^{2} T}{r^{*^{2}} \partial \theta^{*^{2}}}\right]+2 \in\left[\left(\frac{\partial v_{r}^{*}}{\partial r^{*}}\right)^{2}+\right.$

$\left.\left(\frac{\partial v_{\theta}^{*}}{r^{*} \partial \theta^{*}}+\frac{v_{r}^{*}}{r^{*}}\right)^{2}\right]$

نقوم بدمج المعادلتين (2) و (3) باستخدام عمليات رياضية وعليه تصبح معادلة الحركة على النحو الآتي: $\frac{\partial}{\partial \mathrm{t}^{*}}\left[\frac{\partial v_{\theta}^{*}}{\partial \mathrm{r}^{*}}-\frac{\partial v_{r}^{*}}{r^{*} \partial \theta^{*}}\right]-\frac{\partial v_{r}^{*}}{r^{*} \partial \theta^{*}} \frac{\partial v_{r}^{*}}{\partial r^{*}}+v_{r}^{*} \frac{\partial^{2} v_{\theta}^{*}}{\partial r^{*^{2}}}-\frac{v_{r}^{*}}{r^{*}} \frac{\partial^{2} v_{r}^{*}}{\partial \theta^{*} \partial r^{*}}+\frac{v_{\theta}^{*}}{r^{*}} \frac{\partial v_{r}^{*}}{\partial r^{*}}-\frac{v_{\theta}^{*}}{r^{*}} \frac{\partial^{2} v_{r}^{*}}{\partial \theta^{* 2}}-$

$\frac{\partial v_{r}^{*}}{r^{*} \partial \theta^{*}} \frac{\partial v_{\theta}^{*}}{\partial \theta^{*}}+\frac{v_{\theta}^{*}}{r^{*}} \frac{\partial^{2} v_{\theta}^{*}}{\partial \theta^{*} \partial r^{*}}-\frac{\partial\left(v_{\theta}^{*}\right)^{2}}{r^{*} \partial \theta^{*}}=\frac{\mu}{\rho} \nabla^{2}\left[\frac{\partial v_{\theta}^{*}}{\partial r^{*}}-\frac{\partial v_{r}^{*}}{r^{*} \partial \theta^{*}}\right]-\frac{\mu}{\rho} \frac{\partial v_{\theta}^{*}}{r^{* 2} \partial r^{*}}+\frac{\mu}{\rho} \frac{2 \partial^{2} v_{r}^{*}}{r^{*} \partial \theta^{*} \partial r^{*}}+$ $\frac{\mu}{\rho} \frac{\partial v_{r}^{*}}{r^{* 3} \partial \theta^{*}}+\frac{\mu}{\rho} \frac{2 \partial^{2} v_{\theta}^{*}}{r^{2} \theta^{*^{2}}}-g \beta \frac{\partial T}{\partial r^{*}}+\frac{\sigma B_{r} B_{O}}{\rho}\left[-\frac{\partial v_{r}^{*}}{\partial r^{*}}+\frac{\partial v_{\theta}^{*}}{r^{*} \partial \theta^{*}}\right]+\frac{\sigma B_{r}^{2}}{\rho} \frac{\partial v_{\theta}^{*}}{\partial r^{*}}-$ $\frac{\Sigma \mathrm{E}_{\mathrm{O}} \mathrm{B}_{\mathrm{O}}}{\mathrm{P}} \frac{\partial v_{\mathrm{R}}^{*}}{\mathrm{R}^{*} \partial \Theta^{*}}$ 


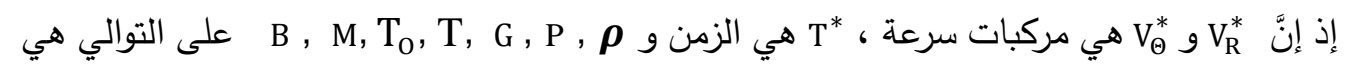

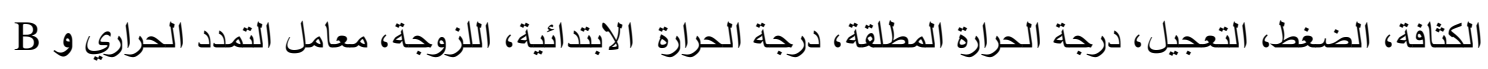
$\sigma, \in=\frac{\mu}{\rho c_{p}}, c_{p}, k, \mathrm{E}=\left(E_{r}, E_{\theta}, E_{z}\right)=\left(0,0, E_{o}\right),=\left(B_{r}, B_{\theta}, B_{z}\right)=\left(B_{r}, B_{o}, 0\right)$ على التوالي هي متجه الحقل المغناطيسي، متجه الحقل الكهربائي، معامل التوصيل الحراري، الحرارة النوعية، معلمة التشتت، اللزوجة الكينماتية. مع الثروط الحدودية الآتية: أ- الثروط الحدودية للحرارة تكون الثروط الحدودية للحرارة عند الجدارين العلوي والسفلي وعلى النحو الآتي :

$\mathrm{T}\left(\mathrm{r}^{*}=L, \theta=\frac{\pi}{2}\right)=T_{h}$

$T\left(r^{*}=L, \theta=\frac{3 \pi}{2}\right)=T_{1}$

إذ إن • التوالي ب- الثروط العدودية للسرعة

$$
\begin{aligned}
& \mathrm{v}_{\mathrm{r}}^{*}\left(\mathrm{r}^{*}, \theta^{*}=\frac{\pi}{2}\right)=\mathrm{v}_{\theta}^{*}\left(\mathrm{r}^{*}, \theta^{*}=\frac{\pi}{2}\right)=0 \\
& \mathrm{v}_{\mathrm{r}}^{*}\left(\mathrm{r}^{*}, \theta^{*}=\frac{3 \pi}{2}\right)=\mathrm{v}_{\theta}^{*}\left(\mathrm{r}^{*}, \theta^{*}=\frac{3 \pi}{2}\right)=0 \\
& \mathrm{v}_{\mathrm{r}}^{*}\left(r^{*}=L, \theta^{*}\right)=\mathrm{v}_{\theta}^{*}\left(r^{*}=L, \theta^{*}\right)=0
\end{aligned}
$$

4- الصيغة اللابعدية للمعادلات المتحكمة في الإحداثيات القطبية: لحل المعادلات المتحكمة مع الثروط الحدودية نحتاج لتحويل المعادلات المتحكمة إلى الصيخ اللابعدية

$$
\begin{aligned}
& u=\frac{u^{*}}{U} \Rightarrow \partial u^{*}=U \partial u \\
& v=\frac{v^{*}}{U} \Rightarrow \partial v^{*}=U \partial v \\
& U=\frac{\alpha}{L} \sqrt{\operatorname{RaPr}=\sqrt{g \beta \Delta T L}} \\
& x=\frac{x^{*}}{L} \Rightarrow \partial x^{*}=L \partial x \\
& y=\frac{y^{*}}{L} \Rightarrow \partial y^{*}=L \partial y \\
& \theta=\frac{T-T_{o}}{T_{h}-T_{o}} \Rightarrow \partial \mathrm{T}=\partial \theta \Delta \mathrm{T} \\
& t=\frac{U t^{*}}{L} \Rightarrow \partial t^{*}=\frac{L}{U} \partial t \\
& \operatorname{Ra}=\frac{\rho g \beta\left(T_{h}-T_{o}\right) L^{3}}{\mu \alpha} \\
& \operatorname{Pr}=\frac{U}{\alpha}=\frac{\mu c_{p}}{k} \\
& \mathrm{EC}=\frac{U^{2}}{c_{p} \Delta T} \\
& \mathrm{H} a=B_{o} L \sqrt{\frac{\sigma}{\mu}}
\end{aligned}
$$

إذ إنَّ : 


$$
\begin{aligned}
& \text { Pr } \\
& \text { [4] Eو Eم Eمد ايكرت EC } \\
& H a \\
& \frac{U \partial v_{r}}{\mathrm{~L} \partial \mathrm{R}}+\frac{U \partial v_{\theta}}{L^{2} r \partial \theta}+\frac{U v_{r}}{L r}=0 \\
& \text { نعوض المقادير أعلاه في المعادلات (1),(4),(5) نحصل على: } \\
& \frac{U v_{\theta}}{L^{2} r^{2}} \frac{U \partial^{2} v_{r}}{L^{2} \partial \theta^{2}}-\frac{U \partial v_{r}}{r^{2} L^{2} L \partial \theta} \frac{U \partial v_{\theta}}{L \partial \theta}+\frac{U v_{\theta}}{L r} \frac{U \partial^{2} v_{\theta}}{L^{2} \partial r \partial \theta}-\frac{U^{2} \partial v_{\theta}^{2}}{L^{3} r^{2} \partial \theta}=\frac{\mu}{\rho}\left[\frac{\partial^{2}}{L^{2} \partial r^{2}}+\frac{\partial}{L^{2} r \partial r}+\right. \\
& \left.\frac{\partial^{2}}{L^{2} r^{2} L^{2} \partial \theta^{2}}\right]\left[\frac{U \partial v_{\theta}}{L \partial r}-\frac{U \partial v_{r}}{L^{2} r \partial \theta}\right]-\quad \frac{\mu}{\rho} \frac{U \partial v_{\theta}}{L^{3} r^{2} \partial r}+\frac{\mu}{\rho} \frac{2 U \partial^{2} v_{r}}{\mathrm{~L}^{2} \mathrm{R}^{2} \mathrm{~L}^{2} \partial \mathrm{R} \partial \Theta}+\frac{\mu}{\rho} \frac{U \partial v_{r}}{L^{3} r^{3} L \partial \theta}+ \\
& \frac{\mu}{\rho} \frac{2 U \partial^{2} v_{\theta}}{L^{3} r^{3} L^{2} \partial \theta^{2}}-g \beta \frac{\Delta T \partial w}{L \partial r}+\frac{\sigma B_{r} B_{O}}{\rho}\left[\frac{U \partial v_{\theta}}{L^{2} r \partial \theta}-\frac{U \partial v_{r}}{L \partial r}\right]+\frac{\sigma B_{r}^{2}}{\mathrm{P}} \frac{\mathrm{U} \partial \mathrm{v}_{\Theta}}{\mathrm{L} \partial \mathrm{R}}-\frac{\sigma E_{o} B_{O}}{\rho} \frac{\partial v_{r}}{L^{2} r \partial \theta} \\
& \frac{\partial \mathrm{W} \Delta \mathrm{T}}{\partial \mathrm{T} \frac{\mathrm{L}}{\mathrm{U}}}+U v_{r} \frac{\partial w \Delta T}{L \partial r}+\frac{U v_{\theta}}{L r} \frac{\partial w \Delta T}{L \partial \theta}=\frac{\mathrm{K}}{\mathrm{PC}_{\mathrm{P}}}\left[\frac{\partial^{2} \mathrm{~W} \Delta \mathrm{T}}{\partial \mathrm{R}^{2} \mathrm{~L}^{2}}+\frac{\Delta T \partial w}{L^{2} r \partial r}+\frac{\Delta T \partial^{2} w}{L^{2} r^{2} L^{2} \partial \theta^{2}}\right]+2 \in \\
& {\left[\left(\frac{U \partial v_{r}}{L r L \partial \theta}\right)^{2}+\left(\frac{U \partial v_{\theta}}{L \partial r}+\frac{U v_{\theta}}{L r}\right)^{2}\right]}
\end{aligned}
$$

بعد تبسيط المعادلات أعلاه نحصل على:

$$
\begin{aligned}
& \frac{\partial v_{r}}{\partial r}+\frac{\partial v_{\theta}}{L r \partial \theta}+\frac{v_{r}}{r}=0 \ldots \ldots(9) \\
& \frac{\partial \Omega}{\partial \mathbf{t}}=\sqrt{\frac{P r}{R a}}\left[\frac{\partial^{2} \Omega}{\partial r^{2}}+\frac{\partial^{2} \Omega}{r^{2} \boldsymbol{\partial \theta}^{2}}+\frac{\partial \Omega}{r \partial r}\right]-\frac{\partial w}{\partial r}+H a^{2} \sqrt{\frac{\mathrm{PR}}{R a}} \quad \Omega
\end{aligned}
$$

إذ إن: $I=\frac{E_{O}}{B_{O}}$

$\left[\frac{\partial \mathrm{w}}{\partial \mathrm{T}}+v_{r} \frac{\partial w}{\partial r}+A_{1} v_{\theta} \frac{\partial w}{\partial \theta}\right]=\frac{1}{\sqrt{\mathrm{R} a P r}}\left[\frac{\partial^{2} \mathrm{w}}{\partial \mathrm{R}^{2}}+A_{2} \frac{\partial w}{\partial r}+A_{3} \frac{\partial^{2} w}{\partial \theta^{2}}\right]+$

$$
\frac{2 E c P r}{\sqrt{\operatorname{RaPr}}}\left[\left(A_{1} \frac{\partial v_{r}}{\partial \boldsymbol{\theta}}\right)^{2}+\left(\frac{\partial \mathbf{v}_{\boldsymbol{\theta}}}{\partial \mathbf{r}}+A_{2} \boldsymbol{v}_{\boldsymbol{\theta}}\right)^{2}\right]
$$

$A_{1}=\frac{1}{L r}, A_{2}=\frac{1}{r}, A_{3}=\frac{1}{L^{2} r^{2}}, A_{4}=\frac{1}{r^{2}}, A_{5}=\frac{1}{L}$

$A_{6}=\frac{1}{r^{3}}, A_{7}=\frac{1}{L r^{2}}, A_{8}=\frac{1}{L r^{3}}, A_{9}=\frac{1}{L^{2} r^{3}}$

إذ إن المعادلات (9)، (10)، (11) هي معادلات المتحكمة بالمسألة بالصيغة اللابعدية في الإحداثيات القطبية.

\section{5- طريقة الاتجاهات المتعاقبة الضمنية (ADI) لحل المسألة:}

نقوم بحل المعادلات بطريقة الاتجاهات المتعاقبة الضمنية (ADI) الملائمة لحل المعادلات التفاضلية الجزئية القطعية المكافئة (Parabolic Partial Differential Equations) وتتميز هذه الطريقة بمميزات عديدة جعلتها من الطرائق المفضلة وذات كفاءة عالية [8] لإيجاد الحل التقريبي لمسألتتا والصيغة العامة لطريقة

$1-\frac{\theta_{i, j}^{*}-\theta_{i, j, n}}{\frac{\Delta t}{2}}=\left(\delta_{x}^{2} \theta_{i, j}^{*}+\delta_{y}^{2} \theta_{i, j, n}\right)$

$2-\frac{\theta_{i, j, n+1}-\theta_{i, j}^{*}}{\frac{\Delta t}{2}}=\left(\delta_{x}^{2} \theta_{i, j}^{*}+\delta_{y}^{2} \theta_{i, j, n+1}\right)$ 
$\theta_{\mathrm{i}, \mathrm{j}}^{*}=\theta_{i, j,\left(\frac{n+1}{2}\right)}, \delta_{x}^{2}=\frac{\partial^{2}}{\partial x^{2}}, \delta_{y}^{2}=\frac{\partial^{2}}{\partial y^{2}}$

إذ نقوم بحل معادلة الطاقة (11) وبعدها نحل معادلة الحركة (10) وعلى النحو الآتي:

أ- أ- حل معادلة الطاقة (11):

1 1 باتجاه محور

$\frac{w_{i, j}^{*}-w_{i, j, n}}{\frac{\Delta t}{2}}+\left(v_{r}\right)_{i, j, n} \frac{w_{i+1, j}^{*}-w_{i-1, j}^{*}}{2(\Delta r)}+A_{1}\left(v_{\theta}\right)_{i, j, n} \frac{w_{i, j+1, n}-w_{i, j-1, n}}{2(\Delta \theta)}=\frac{1}{m}\left[\frac{w_{i+1, j}^{*}-2 w_{i, j}^{*}+w_{i-1, j}^{*}}{(\Delta r)^{2}}+\right.$ $\left.A_{2} \frac{w_{i+1, j}^{*}-w_{i-1, j}^{*}}{2(\Delta r)}+A_{3} \frac{w_{i, j+1, n}-2 w_{i, j, n}+w_{i, j-1, n}}{(\Delta \theta)^{2}}\right]+\frac{\Phi}{m}$

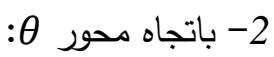

$\frac{w_{i, j, n+1}-w_{i, j}^{*}}{\frac{\Delta t}{2}}+\left(v_{\mathbf{r}}\right)_{i, j, n} \frac{w_{i+1, j}^{*}-w_{i-1, j}^{*}}{2(\Delta r)}+A_{1}\left(v_{\theta}\right)_{i, j, n} \frac{w_{i, j+1, n+1}-w_{i, j}-1, \mathbf{n}+1}{2(\Delta \theta)}=$

$\frac{1}{m}\left[\frac{w_{i+1, j}^{*}-2 w_{i, j}^{*}+w_{i-1, j}^{*}}{(\Delta r)^{2}}+A_{2} \frac{w_{i+1, j}^{*}-w_{i-1, j}^{*}}{2(\Delta r)}+A_{3} \frac{w_{i, j, 1, n+1}-2 w_{i, j, n+1}+w_{i, j}-1, n+1}{(\Delta \theta)^{2}}\right]+\frac{\Phi}{m}$

بعد تبسيط المعادلتين أعلاه نحصل من معادلة (12) على:

$-\mathrm{w}_{\mathrm{i}-1, \mathrm{j}}^{*}+\frac{\left[\frac{2 m}{\lambda}+2\right]}{\left[1-\frac{h A_{2}}{2}+\frac{h m}{2}\left(v_{r}\right)_{i, j, n}\right]} w_{i, j}^{*}-\frac{\left[1+\frac{h A_{2}}{2}-\frac{h m}{2}\left(v_{r}\right)_{i, j, n}\right]}{\left[1-\frac{h A_{2}}{2}+\frac{h m}{2}\left(v_{r}\right)_{i, j, n}\right]} w_{i+1, j}^{*}=$

$\frac{\left[A_{3}+\frac{h A_{1} m}{2}\left(v_{\theta}\right)_{i, j, n}\right]}{\left[1-\frac{h A_{2}}{2}+\frac{h m}{2}\left(v_{r}\right)_{i, j, n}\right]} w_{i, j-1, n}+\frac{\left[\frac{2 m}{\lambda}-2 A_{3}\right]}{\left[1-\frac{h A_{2}}{2}+\frac{h m}{2}\left(v_{r}\right)_{i, j, n}\right]} w_{i, j, n}+$

$\frac{\left[A_{3}-\frac{h A_{1} m}{2}\left(v_{\theta}\right)_{i, j, n}\right]}{\left[1-\frac{h A_{2}}{2}+\frac{h m}{2}\left(v_{r}\right)_{i, j, n}\right]} w_{i, j+1, n}+\frac{\phi h^{2}}{\left[1-\frac{h A_{2}}{2}+\frac{h m}{2}\left(v_{r}\right)_{i, j, n}\right]}$

$\mathrm{i}=1, \ldots \ldots, \mathrm{N}$

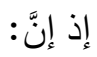

$A(i)=-1, B(i)=\frac{\left[\frac{2 m}{\lambda}+2\right]}{\left[1-\frac{h A_{2}}{2}+\frac{h m}{2}\left(v_{r}\right)_{i, j, n}\right]}, C(i)=-\left(\frac{\left[1+\frac{h A_{2}}{2}-\frac{h m}{2}\left(v_{r}\right)_{i, j, n}\right]}{\left[1-\frac{h A_{2}}{2}+\frac{h m}{2}\left(v_{r}\right)_{i, j, n}\right]}\right)$

$D(i)=\left(\frac{\left[A_{3}+\frac{h A_{1} m}{2}\left(v_{\theta}\right)_{i, j, n}\right]}{\left[1-\frac{h A_{2}}{2}+\frac{h m}{2}\left(v_{r}\right)_{i, j, n}\right]}\right) w_{i, j-1, n}+\left(\frac{\left[\frac{2 m}{\lambda}-2 A_{3}\right]}{\left[1-\frac{h A_{2}}{2}+\frac{h m}{2}\left(v_{r}\right)_{i, j, n}\right]}\right) w_{i, j, n}+$

$\left(\frac{\left[A_{3}-\frac{h A_{1} m}{2}\left(v_{\theta}\right)_{i, j, n}\right]}{\left[1-\frac{h A_{2}}{2}+\frac{h m}{2}\left(v_{r}\right)_{i, j, n}\right]}\right) w_{i, j+1, n}+\frac{\Phi h^{2}}{\left[1-\frac{h A_{2}}{2}+\frac{h m}{2}\left(v_{r}\right)_{i, j, n}\right]}$

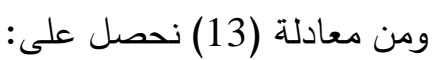

$-\mathrm{w}_{\mathrm{i}, \mathrm{j}-1, \mathrm{n}+1}+\frac{\left[\frac{2 m}{\lambda A_{3}}+2\right]}{\left[1+\frac{h m A_{1}}{2 A_{3}}\left(v_{\theta}\right)_{i, j, n}\right]} w_{i, j, n+1}+\frac{\left[\frac{h m A_{1}}{2 A_{3}}\left(v_{\theta}\right)_{i, j, n}-1\right]}{\left[1+\frac{h m A_{1}}{\left.2 A_{3}\left(v_{\theta}\right)_{i, j, n}\right]}\right.} w_{i, j+1, n+1}=$

$\frac{\left[\frac{1}{A_{3}}-\frac{h m A_{2}}{2 A_{3}}+\frac{h m}{2 A_{3}}\left(v_{r}\right)_{i, j, n}\right]}{\left[1+\frac{h m A_{1}}{2 A_{3}}\left(v_{\theta}\right)_{i, j, n}\right]} w_{i-1, j}^{*}+\frac{\left[\frac{2 m}{\lambda A_{3}}-\frac{2}{A_{3}}\right]}{\left[1+\frac{h m A_{1}}{2 A_{3}}\left(v_{\theta}\right)_{i, j, n}\right]} w_{i, j}^{*}+$

$\frac{\left[\frac{1}{A_{3}}+\frac{A_{1} h}{2 A_{3}}-\frac{h m}{2 A_{3}}\left(v_{r}\right)_{i, j, n}\right]}{\left[1+\frac{h m A_{1}}{2 A_{3}}\left(v_{\theta}\right)_{i, j, n}\right]} w_{i+1, j}^{*}+\frac{\frac{\Phi h^{2}}{A_{3}}}{\left[1+\frac{h m A_{1}}{2 A_{3}}\left(v_{\theta}\right)_{i, j, n}\right]}$

$\mathrm{i}=1, \ldots \ldots, \mathrm{N}$

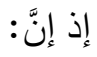




$$
\begin{aligned}
& \mathrm{A}_{1}(i)=-1 \quad, \quad \mathrm{~B}_{1}(i)=\frac{\left[\frac{2 m}{\lambda A_{3}}+2\right]}{\left[1+\frac{h m A_{1}}{2 A_{3}}\left(v_{\theta}\right)_{i, j, n}\right]}, \quad \mathrm{C}_{1}(i)=\frac{\left[\frac{h m A_{1}}{2 A_{3}}\left(v_{\theta}\right)_{i, j, n}-1\right]}{\left[1+\frac{h m A_{1}}{2 A_{3}}\left(v_{\theta}\right)_{i, j, n}\right]} \\
& \mathrm{D}_{1}(i)=\left(\frac{\left[\frac{1}{A_{3}}-\frac{h m A_{2}}{2 A_{3}}+\frac{h m}{2 A_{3}}\left(v_{r}\right)_{i, j, n}\right]}{\left[1+\frac{h m A_{1}}{2 A_{3}}\left(v_{\theta}\right)_{i, j, n}\right]}\right) w_{i-1, j}^{*}+\left(\frac{\left[\frac{2 m}{\lambda A_{3}}-\frac{2}{A_{3}}\right]}{\left[1+\frac{h m A_{1}}{2 A_{3}}\left(v_{\theta}\right)_{i, j, n}\right]}\right) w_{i, j}^{*}+ \\
& \left(\frac{\left[\frac{1}{A_{3}}+\frac{A_{1} h}{2 A_{3}} \frac{h m}{2 A_{3}}\left(v_{r}\right)_{i, j, n}\right]}{\left[1+\frac{h m A_{1}}{2 A_{3}}\left(v_{\theta}\right)_{i, j, n}\right]}\right) w_{i+1, j}^{*}+\frac{\frac{\Phi h^{2}}{A_{3}}}{\left[1+\frac{h m A_{1}}{2 A_{3}}\left(v_{\theta}\right)_{i, j, n}\right]}
\end{aligned}
$$

وهذا يمثل حل معادلة الطاقة في الإحداثيات القطبية

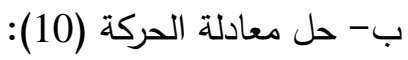
1

$\frac{\Omega_{i, j}^{*}-\Omega_{i, j, n}}{\frac{\Delta t}{2}}=\sqrt{\frac{P r}{R a}}\left[\frac{\Omega_{i+1, j}^{*}-2 \Omega_{i, j}^{*}+\Omega_{i-1, j}^{*}}{(\Delta r)^{2}}+A_{4} \frac{\Omega_{i, j+1, n}-2 \Omega_{i, j, n}+\Omega_{i, j-1, n}}{(\Delta \theta)^{2}}+\right.$

$$
\left.\mathrm{A}_{2} \frac{\Omega_{i+1, j}^{*}-\Omega_{i-1, j}^{*}}{2(\Delta r)}\right]-\frac{w_{i+1, j, n+1}-w_{i+1, j, n+1}}{2(\Delta \theta)}+H a^{2} \sqrt{\frac{\mathrm{PR}}{R a}} \Omega_{i, j, n}
$$

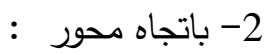

$\frac{\Omega_{i, j, n+1}-\Omega_{\mathrm{i}, j}^{*}}{\frac{\Delta \mathrm{t}}{2}}=\sqrt{\frac{P r}{R a}}\left[\frac{\Omega_{i+1, j}^{*}-2 \Omega_{i, j}^{*}+\Omega_{i-1, j}^{*}}{(\Delta r)^{2}}+A_{4} \frac{\Omega_{i, j+1, n+1}-2 \Omega_{i, j, n+1}+\Omega_{i, j-1, n+1}}{(\Delta \theta)^{2}}+\right.$

$\left.\mathrm{A}_{2} \frac{\Omega_{i+1, j}^{*}-\Omega_{i-1, j}^{*}}{2(\Delta r)}\right]-\frac{w_{i+1, j, n+1}-w_{i-1, j, n+1}}{2(\Delta r)}+H a^{2} \sqrt{\frac{\mathrm{PR}}{R a}} \Omega_{i, j, n+1}$

بعد تبسيط المعادلتين أعلاه نحصل من معادلة (16) على :

$-\Omega_{\mathrm{i}-1, \mathrm{j}}^{*}+\frac{[1+\lambda \beta]}{\left[\frac{\lambda \beta}{2}-\frac{\lambda \beta h \mathrm{~A}_{2}}{4}\right]} \Omega_{i, j}^{*}-\frac{\left[\frac{\lambda \beta}{2}+\frac{\lambda \beta h \mathrm{~A}_{2}}{4}\right]}{\left[\frac{\lambda \beta}{2}-\frac{\lambda \beta h \mathrm{~A}_{2}}{4}\right]} \Omega_{i+1, j}^{*}=\frac{\left[\frac{\lambda \beta A_{4}}{2}\right]}{\left[\frac{\lambda \beta}{2}-\frac{\lambda \beta h \mathrm{~A}_{2}}{4}\right]} \Omega_{i, j-1, n}+$

$\frac{\left[1+\frac{\lambda \beta h^{2}}{2} H a^{2}-\lambda \beta A_{4}\right]}{\left[\frac{\lambda \beta}{2}-\frac{\lambda \beta h A_{2}}{4}\right]} \Omega_{i, j, n}+\frac{\left[\frac{\lambda \beta A_{4}}{2}\right]}{\left[\frac{\lambda \beta}{2}-\frac{\lambda \beta h A_{2}}{4}\right]} \Omega_{i, j+1, n}-\frac{h}{\beta\left[2-4 A_{2} h\right]}\left[w_{i+1, j, n+1}-\right.$

$\left.w_{i-1, j, n+1}\right]$.

$\mathrm{i}=1, \ldots \ldots ., \mathrm{N}$

إذ إنَّ

$A(i)=-1, B(i)=\frac{[1+\lambda \beta]}{\left[\frac{\lambda \beta}{2}-\frac{\lambda \beta h \mathrm{~A}_{2}}{4}\right]}, C(i)=-\frac{\left[\frac{\lambda \beta}{2}+\frac{\lambda \beta h \mathrm{~A}_{2}}{4}\right]}{\left[\frac{\lambda \beta}{2}-\frac{\lambda \beta h \mathrm{~A}_{2}}{4}\right]}$

$\mathrm{D}(i)=\frac{\left[\frac{\lambda \beta A_{4}}{2}\right]}{\left[\frac{\lambda \beta}{2}-\frac{\lambda \beta h A_{2}}{4}\right]} \Omega_{i, j-1, n}+\frac{\left[1+\frac{\lambda \beta h^{2}}{2} H a^{2}-\lambda \beta A_{4}\right]}{\left[\frac{\lambda \beta}{2}-\frac{\lambda \beta h A_{2}}{4}\right]} \Omega_{\mathrm{i}, \mathrm{j}, \mathrm{n}}+$ $\frac{\left[\frac{\lambda \beta A_{4}}{2}\right]}{\left[\frac{\lambda \beta}{2}-\frac{\lambda \beta h \mathrm{~A}_{2}}{4}\right]} \Omega_{i, j+1, n}-\frac{h}{\beta\left[2-4 \mathrm{~A}_{2} h\right]}\left[w_{i+1, j, n+1}-w_{i-1, j, n+1}\right]$

ومن معادلة (17) نحصل على:

$-\Omega_{\mathrm{i}, \mathrm{j}-1, \mathrm{n}+1}+\frac{\left[1+\lambda \beta A_{4}-\frac{\beta \lambda h^{2}}{2} H a^{2}\right]}{\left[\frac{\lambda \beta A_{4}}{2}\right]} \Omega_{\mathrm{i}, \mathrm{j}, \mathrm{n}+1}-\Omega_{i, j+1, n+1}=\frac{\left[\frac{\lambda \beta}{2}-\frac{\lambda \beta h \mathrm{~A}_{2}}{4}\right]}{\left[\frac{\lambda \beta A_{4}}{2}\right]} \Omega_{i-1, j}^{*}+$

$\frac{[1-\lambda \beta]}{\left[\frac{\lambda \beta A_{4}}{2}\right]} \Omega_{i, j}^{*}+\frac{\left[\frac{\lambda \beta}{2}+\frac{\lambda \beta h A_{2}}{4}\right]}{\left[\frac{\lambda \beta A_{4}}{2}\right]} \Omega_{i+1, j}^{*}-\frac{h}{2 \beta A_{4}}\left[w_{i+1, j, n+1}-w_{i-1, j, n+1}\right]$ 
$\mathrm{i}=1, \ldots \ldots, \mathrm{N}$

إذ إنَّ

$\mathrm{A}_{1}(i)=-1, \mathrm{~B}_{1}(i)=\left(\frac{\left[1+\lambda \beta A_{4}-\frac{\beta \lambda h^{2}}{2} H a^{2}\right]}{\left[\frac{\lambda \beta A_{4}}{2}\right]}\right), \mathrm{C}_{1}(i)=-1$

$\mathrm{D}_{1}(i)=\frac{\left[\frac{\lambda \beta}{2}-\frac{\lambda \beta h \mathrm{~A}_{2}}{4}\right]}{\left[\frac{\lambda \beta A_{4}}{2}\right]} \Omega_{i-1, j}^{*}+\frac{[1-\lambda \beta]}{\left[\frac{\lambda \beta A_{4}}{2}\right]} \Omega_{i, j}^{*}+\frac{\left[\frac{\lambda \beta}{2}+\frac{\lambda \beta h A_{2}}{4}\right]}{\left[\frac{\lambda \beta A_{4}}{2}\right]} \Omega_{i+1, j}^{*}-$ $\frac{h}{2 \beta A_{4}}\left[w_{i+1, j, n+1}-w_{i-1, j, n+1}\right]$

وهذا يمثل حل معادلة الحركة في الإحداثيات القطبية.

نستخدم برنامج الحاسوبي بلغة فورتران للحصول على النتائج العددية التي مثلت برسومات توضيحية في الاستنتاجات.

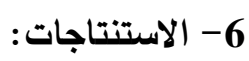

1- لقد لوحظ من حل المعادلات للأنموذج باستخدام طريقة ADI في الإحداثيات القطبية بأنه يمكن الحصول

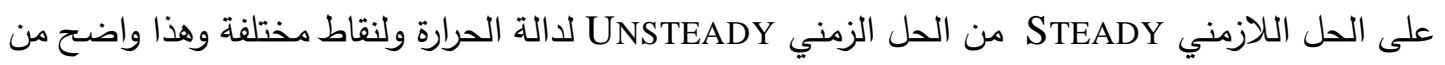

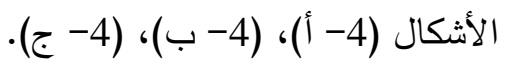

2- تأثير عدد Ra على دالة الحرارة في الإحداثيات القطبية كان واضحاً؛ إذ تراوحت قيم Ra بين (1.5 - (6) 3) وكما

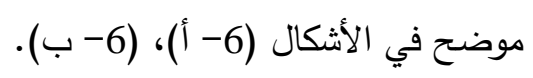

3- لقد لوحظ بأن تأثير عدد Ec ثابت في الإحداثيات القطبية وتراوحت قيم Ec بين (0.02 - 0.04 (0.04) كما مبين

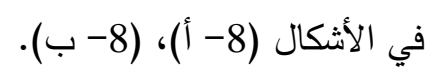

4- أما بالنسبة لدالة الحركة في الإحداثيات القطبية تم الوصول من الحل اللازمني Steady إلى الحل الزمني Unsteady

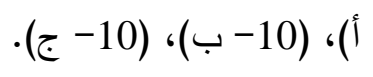

5- إن تأثير عدد Pr على دالة الحرارة في الإحداثيات القطبية كان واضحاً كما موضح في الأشكال (12- أ)،

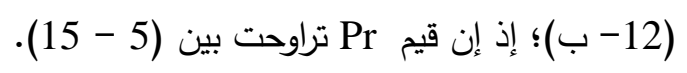

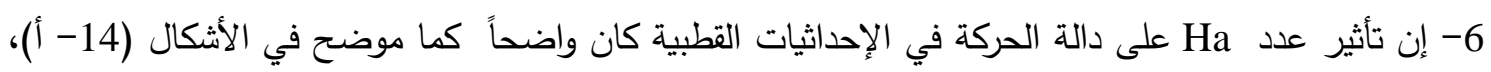

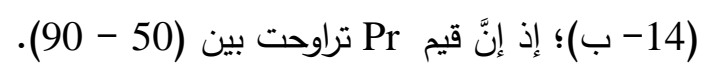

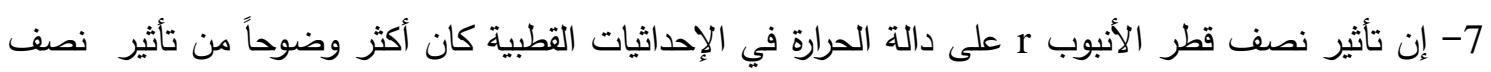

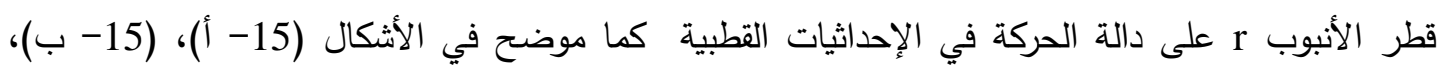
(16- أ)، (16- ب)؛ إذ تراوحت قيم r بين (0.025 -0.25) عند دالة الحركة في الإحداثيات القطبية وتراوحت قيم r بين (0.19-0.25) عند دالة الحرارة في الإحداثيات القطبية. 
الأثكال التوضيحية:

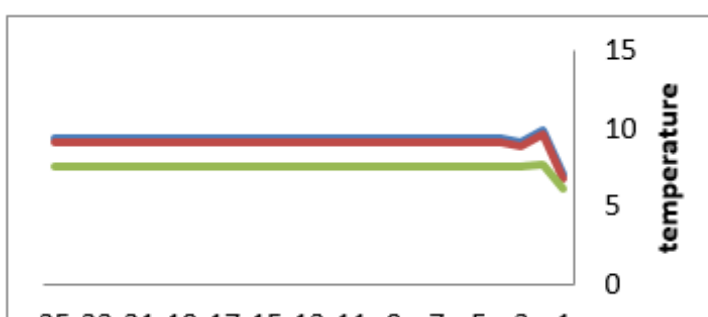

$25232119171513119070503 \quad 1$

iterations

th $(1,5)-\operatorname{th}(5,5)-\operatorname{th}(9,5)$

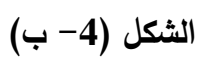

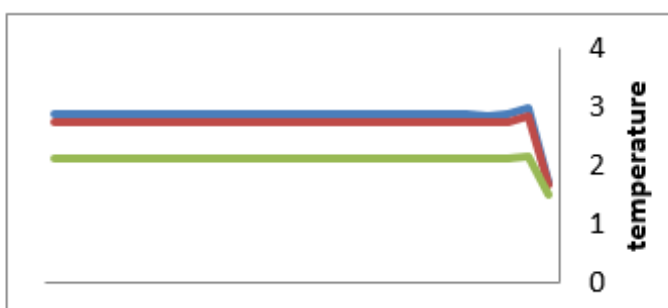

$25232119171513119775 \quad 3 \quad 1$

iterations

th $(1,2)-\operatorname{th}(5,2)-\operatorname{th}(9,2)$

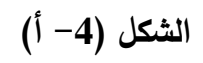

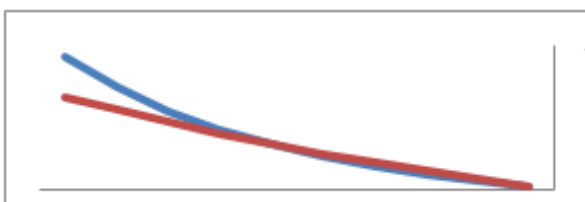

$\begin{array}{llllllllll}10 & 9 & 8 & 7 & 6 & 5 & 4 & 3 & 2 & 1\end{array}$ iterations

th $(2, \mathrm{j}), \mathrm{Ra}=1.5, \mathrm{n}=1$

th $(2, \mathrm{j}), \mathrm{Ra}=1.5, \mathrm{n}=1000$

$$
\text { الشكل (6- (أ) }
$$

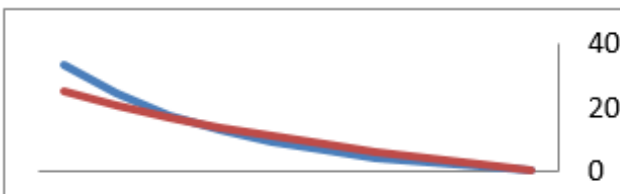

$\begin{array}{llllllllll}10 & 9 & 8 & 7 & 6 & 5 & 4 & 3 & 2 & 1\end{array}$ iterations

th $(8, \mathrm{j}), \mathrm{Ec}=0.02, \mathrm{n}=1$

th $(8, j), E c=0.02, n=1000$

الشكل (8- (أ)

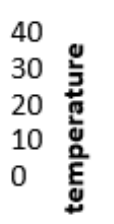

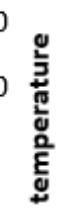
.

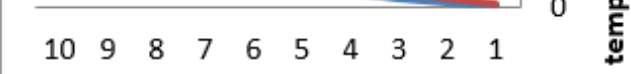
iterations

th $(2, j), R a=3 . n=1$

th $(2, \mathrm{j}), \mathrm{Ra}=3, \mathrm{n}=1000$

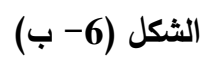



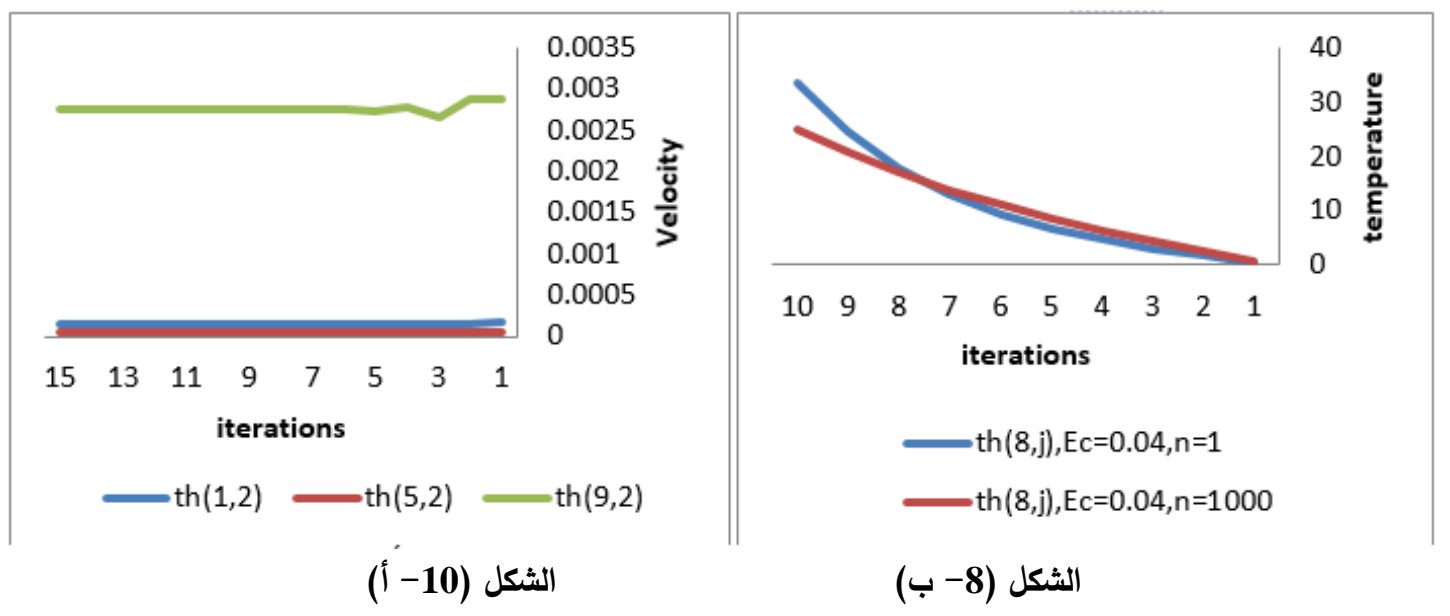

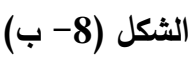

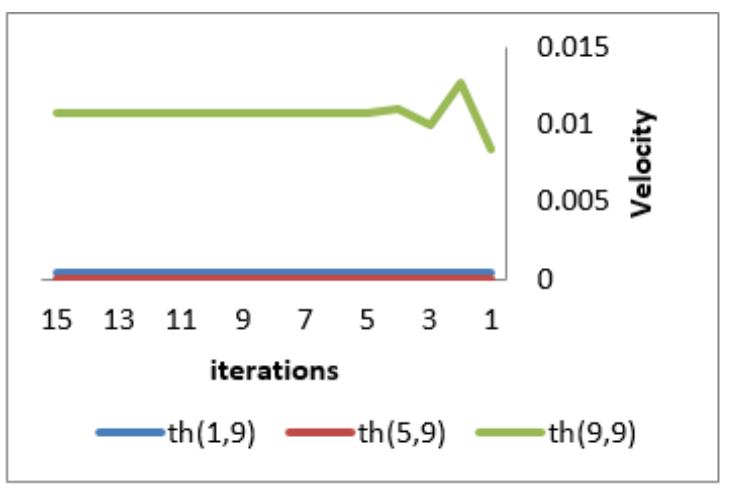

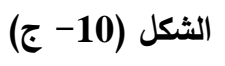

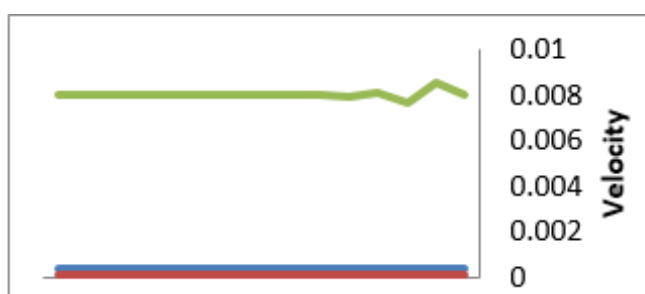

$\begin{array}{llllllll}15 & 13 & 11 & 9 & 7 & 5 & 3 & 1\end{array}$

iterations

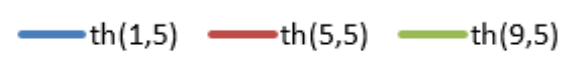

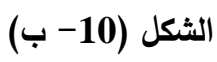

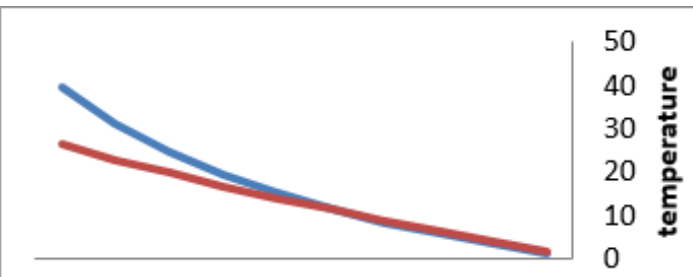

$\begin{array}{llllllllll}10 & 9 & 8 & 7 & 6 & 5 & 4 & 3 & 2 & 1\end{array}$

iterations

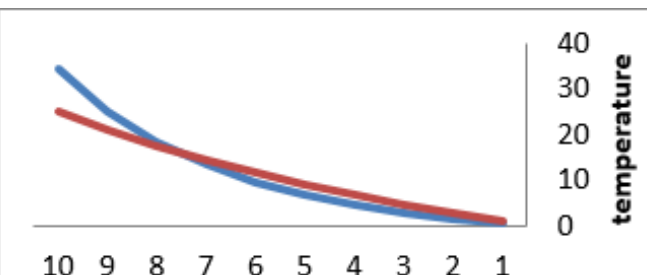

$\begin{array}{llllllllll}10 & 9 & 8 & 7 & 6 & 5 & 4 & 3 & 2 & 1\end{array}$

iterations

th $(5, j), \operatorname{Pr}=15, n=1$

th $(5, \mathrm{j}), \operatorname{Pr}=15, \mathrm{n}=1000$

الثكل (12- (ب)

الثكل (12- أ) 


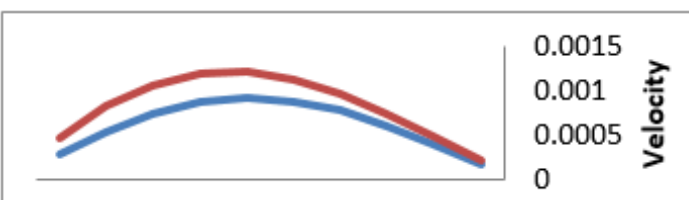

$\begin{array}{llllllllll}10 & 9 & 8 & 7 & 6 & 5 & 4 & 3 & 2 & 1\end{array}$

iterations

th $(7, \mathrm{j}), \mathrm{Ha}=50, \mathrm{n}=1$

th $(7, \mathrm{j}), \mathrm{Ha}=50, \mathrm{n}=1000$

$$
\text { الشكل (14- ب) }
$$

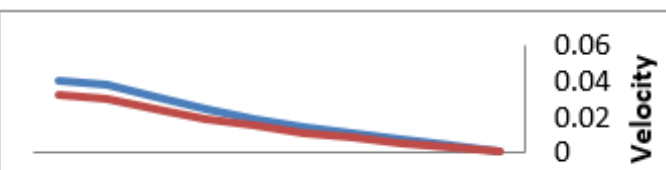

$\begin{array}{llllllllll}10 & 9 & 8 & 7 & 6 & 5 & 4 & 3 & 2 & 1\end{array}$

iterations

th $(10, \mathrm{j}), \mathrm{r}=0.025, \mathrm{n}=1$

th $(10, j), r=0.025, n=1000$

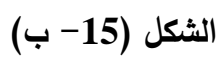

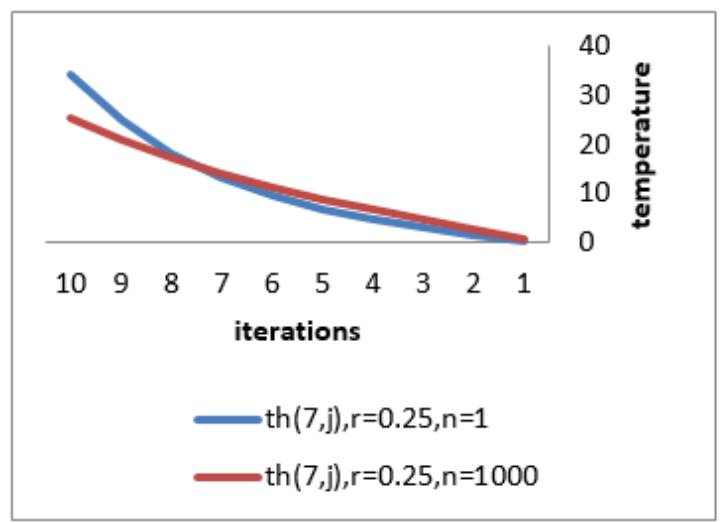

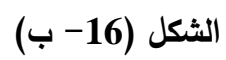

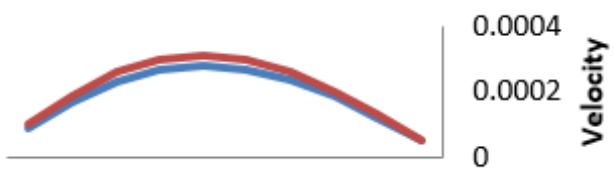

$\begin{array}{llllllllll}10 & 9 & 8 & 7 & 6 & 5 & 4 & 3 & 2 & 1\end{array}$

iterations

th $(7, \mathrm{j}), \mathrm{Ha}=90, \mathrm{n}=1$

th $(7, \mathrm{j}), \mathrm{Ha}=90, \mathrm{n}=1000$

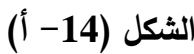

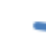

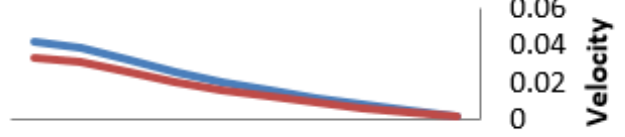

$\begin{array}{llllllllll}10 & 9 & 8 & 7 & 6 & 5 & 4 & 3 & 2 & 1\end{array}$

iterations

th $(10, \mathrm{j}), \mathrm{r}=0.25, \mathrm{n}=1$

th $(10, j), r=0.25, n=1000$

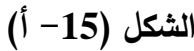

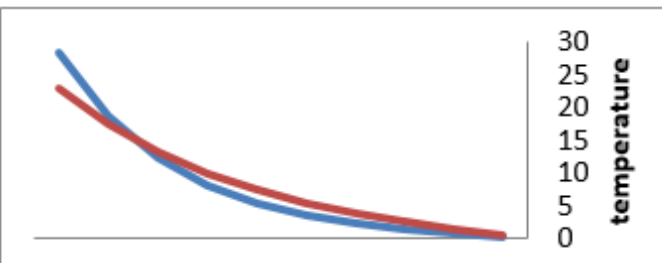

$\begin{array}{llllllllll}10 & 9 & 8 & 7 & 6 & 5 & 4 & 3 & 2 & 1\end{array}$ iterations

th $(7, \mathrm{j}), \mathrm{r}=0.19, \mathrm{n}=1$

th $(7, j), r=0.19, n=1000$

الثكل (16- (أ) 


\section{المصاد}

[1] Bhupendra K. S., Abhay K. JHA , R. C. Chaudhary. ," MHD Forced Flow Of a Conducting Viscous Fluid Through a Porous Medium Induced By an Impervious Rotating Disk". Department of Mathematics, University of Rajasthan 30 Jaipur( 2004), India , E-Mail: rcchaudhary @ rediffmail . com , Received August 22, (2006) .

[2] Davidson, P.A. ," Magnetohydrodynamics in materials processing ". Annu.Rev.Fluid Mech.31,273 300 (1999) .

[3] Hughes, W.F. \& Brighton, J.A., "Fluid Dynamics ". Schaum's Outline Series in Engineering, McGraw-Hill Ltd., USA ( 1967) .

[4] Jumaa , A.M. , "convection in an inclined fluid layer ". Ph.D. Thesis, University of Pune, India (2001) .

[5] J.V. Ramana Muthy , G. Nagaraju , K.S. Sai. ," Numerical Solution for MHD Flow of Micro Polar Fluid Between Two Concentric Rotating Cylinders With Porous Lining ". ISSN 1749-3889 (print), 1749-3897 (online), International Journal of Nonlinear Science, Vol.13, No.2,pp.183-193 (2012) .

[6] Lee, D. \& Choi, H. ," Magnetohydrodynamic turbulent flow in a channel at low magnetic Reynolds number ". J. Fluid Mech. 439, 367394 (2001) .

[7] Mohammed al-khawaja , Mohamed selmi , "Finite difference solutions of MFM square duct flow with heat transfer using matlab program" . Qatar university , Doha , state of Qatar (2010) .

[8] Morton, K. W. \& Mayers, D. F. ," Numerical Solution of Partial Differential Equations ". $2^{\text {nd }}$ Ed. Cambridge University Press , UK (2010) .

[9] Nur Hosain, Abdullah, Md.Mustafa ," Magnetohydrodynamic (MHD) Laminar Free Convective Flow Across a Horizontal Circular Cylinder ". Proceeding of International Conference on Mechanical Engineering (ICME2007) 29-31 December 2007, Dhaka, Bangladesh (2007) .

[10] Patil Vishwambhars and Timol M. G. ," Similarity Solutions of a Class of Laminar Three- Dimensional Magnetohydrodynamic Boundary Layer Equation of Power Law Fluids ". Int. J. open Problems Compt. Math., Vol. 4, No. 2, June 2011 ISSN 1998-6262 Copyright (C) ICSRS Publication, www. i-csrs.org (2011).

[11] Thomas, B.G \& Zhang, L.," Mathematical modeling of fluid flow in continuous casting ". a review. ISIJ Intern 41 (10), 11811193 (2001) .

[12] Von Ammon, W., Gelfgat, Y., Gorbunov, L., Muhlbauer, A., Muiznieks, A., Makarov , Y., Virbulis, J. \& Muller, G.," Application of magnetic fields in industrial growth of silicon single crystals ". In the $15^{\text {th }}$ Riga and $6^{\text {th }}$ Pamier Conference on Fundamental and Applied MHD Modeling of MHD turbulence, vol. I, pp. 41 54.Riga,Latvia (2005) .

[13] Yang, Y.," Natural convective Flow and Heat Transfer in Vertical and Inclined Glazing Cavities ". M.Sc. Thesis, University of Massachusettes (2003) . 